\title{
Context-dependent modulation of cognitive control involves different temporal profiles of fronto-parietal activity
} \author{
Eva Van den Bussche ${ }^{a}$, Tom Verguts ${ }^{c}$ \\ ${ }^{a}$ Brain and Cognition, Faculty of Psychology and Educational Sciences, KU Leuven, 3000, Leuven, Belgium \\ ${ }^{\mathrm{b}}$ Department of Experimental and Applied Psychology, Vrije Universiteit Brussel, 1050, Brussels, Belgium \\ ${ }^{c}$ Department of Experimental Psychology, Ghent University, 9000, Ghent, Belgium
}

Bart Aben $^{\text {a,b,c, }}$, Cristian Buc Calderon ${ }^{\mathrm{c}}$, Laurens Van der Cruyssen ${ }^{\mathrm{b}}$, Doerte Picksak ${ }^{\mathrm{b}}$,

\section{A R T I C L E I N F O}

\section{Keywords:}

Attention

Cognitive effort

fMRI

Proportion congruency

Proactive control

Reactive control

\begin{abstract}
A B S T R A C T
To efficiently deal with quickly changing task demands, we often need to organize our behaviour on different time scales. For example, to ignore irrelevant and select relevant information, cognitive control might be applied in reactive (short time scale) or proactive (long time scale) mode. These two control modes play a pivotal role in cognitive-neuroscientific theorizing but the temporal dissociation of the underlying neural mechanisms is not well established empirically. In this fMRI study, a cognitive control task was administered in contexts with mainly congruent (MC) and mainly incongruent (MI) trials to induce reactive and proactive control, respectively. Based on behavioural profiles, we expected cognitive control in the MC context to be characterized by transient activity (measured on-trial) in task-relevant areas. In the MI context, cognitive control was expected to be reflected in sustained activity (measured in the intertrial interval) in similar or different areas. Results show that in the MC context, on-trial transient activity (incongruent - congruent trials) was increased in fronto-parietal areas, compared to the MI context. These areas included dorsolateral prefrontal cortex (dlPFC) and intraparietal sulcus (IPS). In the MI context, sustained activity in similar fronto-parietal areas during the intertrial interval was increased, compared to the MC context. These results illuminate how context-dependent reactive and proactive control subtend the same brain areas but operate on different time scales.
\end{abstract}

\section{Introduction}

When our GPS and the road signs point us in opposite directions, we need to overcome automatic tendencies (e.g., adhering to an outdated GPS) in favour of more appropriate responses (e.g., following the road signs). Exerting such cognitive control is crucial in everyday life but also cognitively demanding (Kool et al., 2010; Shenhav et al., 2017; Vassena et al., 2014; Verguts et al., 2015). Adaptive allocation of cognitive control therefore requires a balance between exerting a sufficient amount (to solve hard tasks), but not too much (to spare costly cognitive effort). As a result, cognitive control is best applied sparsely and should only be sustained when it is frequently needed (Botvinick et al., 2001; Ridderinkhof, 2002; Braver et al., 2007; Braver, 2012; Jiang et al., 2014).

One way to minimize cognitive expenses while maintaining acceptable performance levels is by applying cognitive control on different time scales. This is mirrored in the way incongruent (i.e., difficult) trials are handled in typical cognitive control tasks. Examples of incongruency can be found in the Stroop task (Stroop, 1935), where word colour must be ignored in favour of word meaning, and in the flanker task (Eriksen and Eriksen, 1974), where one should respond to the central target and ignore the flankers. When incongruent trials are rare, reactive control is thought to be active, meaning that control operates in a just-in-time regime (short time scale). Reactive control is thought to be implemented by transient reactivation of task-relevant brain areas (Braver, 2012; Bugg and Crump, 2012; Logan and Zbrodoff, 1979). On tasks with mainly incongruent trials, this transient strategy could lead to frequent errors and delays. Here, a proactive control mode is optimal, operating on a long time scale.

It has been proposed that the brain regions activated during proactive control show anatomical or functional overlap with those activated during reactive control. For example, similar or closely related regions in lateral PFC have been suggested to be involved in reactive and proactive control depending on the specifics of task demands (Braver, 2012; De Pisapia and Braver, 2006). First preliminary support for this

\footnotetext{
* Corresponding author. Brain \& Cognition, Faculty of Psychology and Educational Sciences, KU Leuven, Tiensestraat 102, 3000, Leuven, Belgium.

E-mail address: bart.aben@kuleuven.be (B. Aben).
} 
context-dependent two-mode (i.e., reactive and proactive) theory of cognitive control came from studies that compared cognitive control between mainly congruent (MC) and mainly incongruent (MI) contexts. In MC contexts, transient (e.g., on-trial) activity in anterior cingulate cortex (ACC) and other fronto-parietal areas is typically higher on incongruent than on congruent trials (Carter et al., 2000; De Pisapia and Braver, 2006; Grandjean et al., 2012; Jaspar et al., 2016). This is taken as an instance of reactive control and results in slow response times (RTs) and low accuracy on incongruent compared to congruent trials. In MI contexts, the same areas are often found to be activated but the difference between incongruent and congruent trials is less strong or even absent (Carter et al., 2000; De Pisapia and Braver, 2006; Grandjean et al., 2012; Marini et al., 2016). This is interpreted as an indicator of sustained control across all trials (congruent and incongruent) in proactive control mode, reducing or eliminating neural and behavioural differences between trial types.

Another way of indexing time scale differences in cognitive control is through hybrid fMRI designs that combine block- and event-related responses (Petersen and Dubis, 2012; Visscher et al., 2003). Here, reactive control is indexed by transient activity on the trial level and proactive control by sustained activity on the block level. Using these designs, the same fronto-parietal areas have been found active in transient and sustained manners, depending on whether the context was MC or MI (Braver et al., 2003; Marini et al., 2016). However, similar designs have also led to claims that two control modes indeed exist but that they not only differ in temporal activation profile but also comprise different brain areas (e.g., Olsen et al., 2010; Seeley et al., 2007). For example, Dosenbach et al. (2008) suggested that transient adjustments in control are initiated by a fronto-parietal network including lateral prefrontal and superior parietal cortices. Sustained control, on the other hand, was supported by a cingulo-opercular network comprising dorsal ACC and the anterior insula. Consistent with this dual-network perspective on cognitive control, Wilk et al. (2012) also showed that transient and sustained activity can arise from different brain areas. However, in this case, the ACC, anterior insula, and inferior parietal cortex showed transient activity, while sustained activity was found in medial superior frontal gyrus.

In the current study, we adapted the event-related fMRI paradigm to investigate time scale differences in cognitive control. Typically, eventrelated paradigms measure on-trial activation and are therefore informative about transient, but not sustained activation. To address this issue, we used activity measured in intertrial intervals as a proxy for sustained activation to identify how cognitive control operates in contexts with mainly congruent or mainly incongruent trials. Thus, "active" flanker trials were interleaved with "blank" trials that consisted of a prolonged fixation cross, which allowed measuring context-dependent intertrial activation (Horga et al., 2011). This method offers a novel perspective on two-mode theories about cognitive control and tests whether cognitive control can indeed be allocated with different temporal profiles (reactively and proactively) depending on the context (MC or MI), and whether this involves similar or different brain areas. The blank trials have the additional advantage that they allow measuring

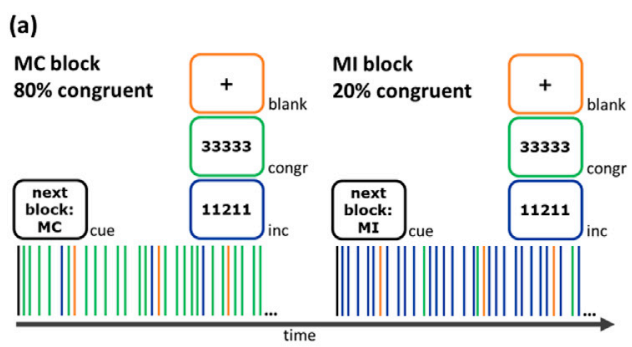

activation independent of differences in stimulus-response contingencies, trial difficulty, motor response, accuracy, or response time (Grinband et al., 2011; Schmidt and Besner, 2008).

If context-dependent cognitive control is indeed differently implemented through transient and sustained neural activation, then two neural patterns can be predicted. First, increased on-trial (i.e., transient) activity in typical cognitive control areas, such as dorsolateral prefrontal cortex (dlPFC), dorsal anterior cingulate cortex (dACC), and parietal cortices (Corbetta and Shulman, 2002; Dosenbach et al., 2008; Niendam et al., 2012) is expected on incongruent compared to congruent trials in the MC block but not in the MI block. Second, increased blank-trial activation is expected in the MI block compared to the MC block, reflecting the sustained recruitment of cognitive control in the MI block. Transient and sustained control activation might be located in similar (Braver, 2012; Kerns et al., 2004; Marini et al., 2016) or different (Dosenbach et al., 2008; Olsen et al., 2010; Wilk et al., 2012) brain areas.

\section{Materials and methods}

\subsection{Participants}

Twenty-one healthy native Dutch-speaking participants gave written informed consent to participate. The study was approved by the ethics committee of the Ghent University Hospital. All participants reported no history of psychiatric or neurological disorder, were right-handed, had normal or corrected-to-normal vision, and were rewarded with 30 Euros in exchange for their participation. In addition, a 25 Euros gift coupon was awarded to the best performing participant (as measured by a combined index of RT and accuracy), to increase motivation. One participant was excluded from the analysis because of a technical failure, leaving twenty participants included for statistical analysis (13 females, 7 males, $M$ age $=23.32, S D=2.29$, age range $=21-28$ ).

\subsection{Behavioural task}

Participants performed a numerical Eriksen flanker task with the numbers 1, 2, 3, and 4. Stimuli consisted of a central target number, flanked by four identical distractor numbers on both sides (e.g., 11311; see Fig. 1a). Participants were instructed to respond to the central target number accurately and as fast as possible by pressing the corresponding button with their left middle finger (1), left index finger (2), right index finger (3), or right middle finger (4). Stimuli $\left(3^{\circ}\right.$ wide and $1^{\circ}$ high) were displayed until response in black against a grey background and separated by a pseudo-exponential intertrial interval of $1200-7200 \mathrm{~ms}$ (average $=3000 \mathrm{~ms}$ ) during which a black fixation cross was displayed centrally. Two different trial types were presented, namely active and blank trials. Active trials could be congruent (i.e., trials with identical target and flankers, e.g., 11111) or incongruent (i.e., trials with different target and flankers, e.g., 11211). All combinations of target and flanker numbers appeared with equal probability on incongruent trials. During blank trials, the fixation cross was
(1)

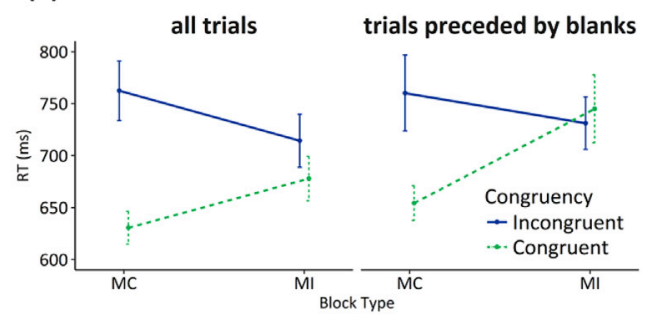

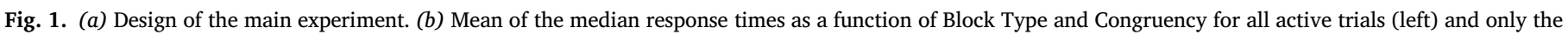
active trials preceded by a blank (right). MC $=$ mainly congruent, $\mathrm{MI}=$ mainly incongruent. Error bars represent standard errors. $* * * p<0.001, * * p<0.01$. 
presented on screen for $700 \mathrm{~ms}$ after the intertrial interval (i.e., a fixation cross remained on screen for an additional $700 \mathrm{~ms}$ and no flanker trial was shown). Importantly, because active and blank trials were separated by a jittered intertrial interval, activation on blank trials could be differentiated from activation on the preceding active trials. To the subject, the blank trials did not differ from regular intertrial intervals, since both consisted of a fixation cross.

\subsection{Procedure}

The experiment consisted of a prescanning training outside the scanner and the main session inside the scanner. The whole experiment took about $1 \mathrm{~h} 15 \mathrm{~min}$.

\subsubsection{Main experiment}

Two types of blocks of 68 trials each were presented, with different proportions of congruent trials (see Fig. 1a). In the MC block, 48 trials were congruent, 12 were incongruent, and eight were blank, resulting in a congruent:incongruent ratio of 80:20. This ratio was reversed in the MI block with 12 congruent, 48 incongruent and eight blank trials (Horga et al., 2011). Participants were not informed about the occurrence of blank trials but were told that the duration of the fixation cross could vary across trials and that they should stay focused during these intervals. Four MC and four MI blocks were created, resulting in eight blocks that were presented in random order. The task was administered in two consecutive runs with four blocks each. Each block was preceded by a 30s break period.

Trial presentation within a block was random with two constraints. First, in each block, blanks were equally often preceded by a congruent trial and an incongruent trial. Second, each block started with four items representative for the block type (e.g., four congruent items in the MC block). This served to induce the appropriate cognitive control mode at the beginning of the block, to optimize effects on early occurring blanks in that block. For the same reason, participants were cued whether the upcoming block would be MC or MI. The difference between the block types was taught in a separate training session outside the scanner before the start of the experiment (see below), which also served as a practice session. Another ten practice trials were presented inside the scanner before the main experiment to make participants acquainted with the experimental setting.

\subsubsection{Prescanning training}

The prescanning training had two goals: 1) to familiarize participants with the task and the stimulus-response mappings, and 2) to make participants understand the difference between MC and MI blocks. Participants performed several blocks of $50 \%$ congruent practice trials with accuracy feedback. Practice ended as soon as the participant completed a block of 20 trials with $80 \%$ accuracy. Next, participants received verbal explanation on the difference between congruent and incongruent trials. To the participants, congruent trials were labelled "identical" and incongruent trials "conflicting" to avoid the rather abstract labels "congruent" and "incongruent". After that, participants performed several 20-trial MC and MI blocks and were asked to identify each block as MC (i.e., "mostly identical trials") or MI (i.e., "mostly conflicting trials"). This procedure was terminated after the correct identification of four consecutive blocks.

\subsection{Behavioural data analyses}

Two-by-two repeated-measures analyses of variance (ANOVA) with the factors Block Type (MC, MI) and Congruency (congruent, incongruent) were used to analyse the median RT and mean error rate of all trials and trials following blank trials. The following trials were excluded for the RT analysis: trials with RTs faster than $250 \mathrm{~ms}(0 \%)$ or slower than $2500 \mathrm{~ms}$ (1.7\%), error trials (1.9\%) and trials following errors (1.9\%).

\section{5. fMRI acquisition}

Images were collected by means of a $3 \mathrm{~T}$ Magnetom Trio MRI scanner system (Siemens Medical Systems, Erlangen, Germany), with a 32-channel radiofrequency head coil. Participants perceived stimuli projected onto a screen at the extremity of the magnet bore through a mirror mounted on the head coil. Stimulus presentation was controlled by EPrime 2.0 (Psychology Software Tools, 2012). First, a high-resolution T1-weighted structural scan (MP-RAGE) was conducted followed by two functional runs using a gradient-echo echo-planar pulse sequence. Functional images consisted of 30 axial slices ( $4 \mathrm{~mm}$ thick; $1 \mathrm{~mm}$ skip), with $\mathrm{TR}=2 \mathrm{~s}, \mathrm{TE}=33 \mathrm{~ms}$, and $3.5 \times 3.5 \times 4.0 \mathrm{~mm}$ in-plane resolution.

\section{6. fMRI data analysis}

\subsection{1. fMRI data pre-processing}

The fMRI data were pre-processed and analysed using SPM12 (http:// www.fil.ion.ucl.ac.uk/spm/software/spm12). Functional data were corrected for differences in acquisition times between slices for each wholebrain volume, realigned within and across runs, and co-registered with each participant's anatomical scan. The functional data were then segmented and spatially normalized to a standard MNI space $(2 \mathrm{~mm}$ isotropic voxels). Normalized data was spatially smoothed (6 mm fullwidth at half-maximum, FWHM) using a Gaussian kernel filter. Six motion parameters were estimated using the Artifact Detection Tool software package (ART; https://www.nitrc.org/projects/artifact_detect). These parameters were used to check for outlier scans, which were identified in the temporal differences series by between-scan differences using the following criteria: Z-threshold: $3.0 \mathrm{~mm}$; scan to scan movement threshold: $0.5 \mathrm{~mm}$; rotation threshold: 0.02 radians. Outliers were omitted from statistical analysis by including a single regressor for each outlier. Finally, using ART, no correlations between motion and experimental design, and between global mean signal and experimental design were identified.

\subsubsection{Individual-level analysis}

Using a general linear model, blood-oxygenation-level dependent (BOLD) responses for all participants were modelled at each voxel. The single-subject analysis was conducted per run and included regressors for each of the events resulting from the within-subjects design involving Block Type (MC, MI) and Congruency (congruent, incongruent) for active trials (i.e., MC_C, MC_I, MI_C, MI_I) and Block Type (MC, MI) for blank trials (i.e., MC_blank, MI_blank), leading to a total of six regressors of interest. In addition, regressors for cues, errors, and previous errors were included as regressors of non-interest. The six motion parameters and outlier regressors identified with ART were included as nuisance regressors. All regressors were time-locked at the onset of the trial. Active and blank trials were analysed in one model with different basis functions that each capitalized on the properties of the active and the blank trials, thus maximizing statistical power. The active trials were convolved with the canonical hemodynamic response function (HRF) and its time derivative, while the blank trials were convolved with a finite impulse response (FIR) function. The canonical HRF keeps estimator variability low at the cost of some bias, compared to the FIR function. The FIR model was chosen for blanks because of the atypical (non-task) nature of the trials. This strategy allowed for more flexibility to capture the hemodynamic response function (i.e., less bias), at the cost of increased estimator variability (Poldrack et al., 2011). The FIR was estimated across 12 time points, $2 \mathrm{~s}$ apart (i.e., the TR), starting at the event onset and ending $22 \mathrm{~s}$ later. A high-pass filter of $0.008 \mathrm{~Hz}$ was applied and temporal autocorrelations were accounted for using the default first order auto-regressive, or AR(1), model.

Importantly, since all (i.e., active and blank) trials were preceded by a jittered intertrial interval, correlations between active and blank trials were kept low. Indeed, the average correlation between active and blank trial regressors was 0.01 (range: $-0.19-0.21$ ). 


\subsubsection{Group-level analysis of active trials}

One-sample $t$-contrasts for the active trials (i.e., MC_C, MC_I, MI_C, and MI_I) averaged over both runs were taken to a second-level random effects model. We first checked for differences between blocks $\left(\left[\mathrm{MI} I+\mathrm{MI}_{-} \mathrm{C}\right]>\left[\mathrm{MC} \mathrm{I}_{-}+\mathrm{MC} C\right]\right)$ and congruency levels ([MC_I + MI_I $>$ [MC_C + MI_C). To assess which areas are active during cognitive control in the MC block, we next contrasted incongruent and congruent trials in MC blocks (i.e., MC_I > MC_C). The same events were contrasted in the MI block (i.e., MI_I > MI_C). These two analyses were in turn contrasted (i.e., [MC_I $>$ MC_C] $>$ [MI_I $>$ MI_C]) to test for differences in transient activity in the MC versus the MI block. This contrast indirectly tests the prediction that cognitive control in the MI block is characterized by proactive or sustained control across all trials, leading to smaller or absent differences in transient activation between incongruent and congruent trials in the MI block.

Comparisons of interest were tested using two-sample $t$-tests on the group level. Results are reported at a voxel-based threshold corrected for familywise error of multiple comparisons (FWE voxel-wise correction, i.e., $p_{F W E}<0.05$ ). In addition, we imposed a minimum cluster extent of 10 voxels. For visualization of results, statistical maps were projected onto a cortical surface with the use of Surf Ice (https://www.nitrc.org/ projects/surfice).

\subsubsection{Group-level analysis of blank trials}

First, functional ROIs were created using the MarsBar toolbox (http:// marsbar.sourceforge.net/) based on the outcome of the group-level analyses of the active trials. These ROIs involved a sphere of $8 \mathrm{~mm}$ radius around the group-level coordinates of the peak activity of contrast MC_I > MC_C. To assess the second hypothesis that areas that are transiently activated in MC blocks are also active in a sustained way in MI blocks, we compared ROI activity between blanks on MI and MC blocks (i.e., MI_blank > MC_blank). This comparison tests the prediction that task-relevant activation returns to baseline between trials in the MC block but remains high throughout the MI block. Note that this ROI analysis is performed on different trial types than the active-trial analysis (i.e., blanks). Moreover, the contrast of interest is opposite to the one that provided the ROIs, thus precluding double dipping.

FIR functions were averaged across voxels and runs within the ROIs for each participant. Cluster-based non-parametric permutation tests were used for testing the averaged FIR regressor estimates (beta's) for the contrast MI_blank > MC_blank (Maris and Oostenveld, 2007). This method controls for multiple testing (i.e., the FIRs must be compared at 12 different time-points) by taking into account the temporal structure of the data (i.e., the fact that the 12 bins are not independent but have a temporal dependence structure). It allows to investigate the full FIR model, while controlling for multiple testing and without pre-specifying the temporal locus of effect. First, paired sample $t$-tests were performed comparing the regressor estimates at each timepoint between MI and MC blocks. The absolute $t$-values of adjacent timepoints that exceeded the critical $t$-value for a two-tailed $t$-test with 19 degrees of freedom (i.e., $t<-2.09$ and $t>2.09$ ) were summed to a maximum observed cluster- $t$. Second, this procedure was repeated after randomly permuting the condition labels (i.e., "MC" and "MI") of each participant 10,000 times, resulting in a null distribution of permutation maximum cluster-t's. Finally, a $p$-value was obtained by testing the observed cluster-t against the null distribution. Statistical significance was inferred when the observed cluster- $t$ exceeded the 95th percentile of this null distribution.

In addition, we also explored blank-trial activation on the wholebrain level. To restrict the number of tested time bins, we calculated blank-trial activation in five time bins ranging from 4 to $12 \mathrm{~s}$ after blank onset (i.e., bins 3 to 7). Next, we compared blank-trial activation between MI and MC blocks in each of the five time bins individually. These results were obtained with at a voxel-based threshold corrected for FWE of multiple comparisons (voxel-wise correction, i.e., $p_{F W E}<0.05$ ). In addition, a minimum cluster extent of 10 voxels was imposed.

\section{Results}

\subsection{Behavioural results}

A repeated-measures ANOVA was conducted on RTs with the withinsubject factors Block Type (MC, MI) and Congruency (congruent, incongruent). Effects are displayed in Fig. $1 \mathrm{~b}$. The results showed a main effect of Congruency, $F(1,19)=87.78, p<0.001$, but not of Block Type, $F(1,19)<0.01, p=0.98$. Responses were slower on incongruent trials (738 ms) than on congruent trials ( $654 \mathrm{~ms}$ ), indicating a congruency effect (CE). A proportion congruency effect (PCE) was also found, as evidenced by the interaction between Block Type and Congruency, $F(1$, $19)=22.01, p<0.001$. The CE was larger in the MC block (132 ms) than in the MI block ( $37 \mathrm{~ms}$ ), showing a behavioural dissociation between reactive and proactive control. Similar effects were obtained when the analysis was restricted to the trials preceded by blanks. A main effect of Congruency was found $F(1,19)=12.06, p=0.003$, but not of Block Type, $F(1,19)=2.56, p=0.13$. Block Type and Congruency also interacted (i.e., a PCE), $F(1,19)=11.03, p=0.004$ (see Fig. 1b).

A similar repeated measures ANOVA was conducted on error rates. Overall, participants committed few errors (1.9\%). The ANOVA revealed a main effect of Block Type, $F(1,19)=12.28, p=0.002$, with larger error rates on MC blocks (2.4\%) than on MI blocks (1.5\%), and a main effect of Congruency, $F(1,19)=9.31, p=0.007$, with more errors on incongruent trials $(2.6 \%)$ than on congruent trials $(1.4 \%)$. No interaction between Block Type and Congruency was found, $F(1,19)=0.28, p=0.60$. Analyses restricted to trials preceded by blanks showed a marginally significant main effect of Block Type, $F(1,19)=4.13, p=0.056$, with larger error rates on MC blocks (2.4\%) than on MI blocks (0.9\%). No effect of Congruency, $F(1,19)=1.64, p=0.22$ was found, nor an interaction between Block Type and Congruency, $F(1,19)=0.12, p=0.73$.

\section{2. fMRI results}

\subsubsection{Larger transient activity on active trials during reactive control}

First, main effects of Block Type and Congruency were assessed. Comparison of the two block types (MI > MC), independent of congruency, yielded no significant activation. Comparison of incongruent and congruent trials, independent of Block Type, revealed larger activation of intraparietal sulcus during incongruent trials (i.e., a general congruency effect; Table 1).

Next, incongruent and congruent trial activation was compared within each block type (MC and MI). It was hypothesized that incongruent trials elicit stronger transient activation in cognitive control areas in the MC blocks (i.e., on MC_I > MC_C). Accordingly, activation was observed in a network of fronto-parietal areas, including the bilateral intraparietal sulcus (IPS), the left inferior frontal gyrus (IFG), the bilateral middle frontal gyrus (MFG), the right dlPFC, and the left presupplementary motor area (pre-SMA; see Table 1 and Fig. 2a). Contrasting incongruent and congruent trials in the MI block (MI_I > MI_C) was expected to reveal smaller or no transient activation compared to the same contrast in the MC block. Indeed, no voxels survived the statistical threshold in this contrast.

To ensure that the obtained effects reflect differences in cognitive control mode and are not due to a difference in surprise, we also compared congruent trials to incongruent trials in the MI block. Note that congruent trials in MI blocks are equally surprising as incongruent trials in MC blocks. However, cognitive control is only expected to be activated on incongruent trials in the MC block. This contrast (MI_C $>$ MI_I) revealed no activation, ruling out surprise effects.

Finally, cognitive control in MC and MI block was compared directly (i.e., $\left[\mathrm{MC} \mathrm{I}_{-}>\mathrm{MC} \mathrm{C}_{-}\right]>[\mathrm{MI} \mathrm{I}>\mathrm{MI} \mathrm{C}]$ ). This contrast revealed stronger activation on the MC block in areas identified above, including the bilateral IPS, left IFG, and left MFG (Table 2 and Fig. 2b). The reversed contrast (i.e., [MI_I $>$ MI_C] $>$ [MC_I $>$ MC_C]) did not reveal any activation. This suggest that on a transient level, the fronto-parietal network 
Table 1

Peak voxel activation in incongruent (minus congruent) trials (i.e., the general congruency effect, I > C) across MC and MI blocks and in the MC and MI blocks separately.

\begin{tabular}{|c|c|c|c|c|c|c|c|}
\hline \multirow[t]{2}{*}{ Region } & \multirow[t]{2}{*}{ Side } & \multirow{2}{*}{$\begin{array}{l}\text { Cluster } \\
\text { size }\end{array}$} & \multirow{2}{*}{$\begin{array}{l}Z- \\
\text { score }\end{array}$} & \multirow[t]{2}{*}{$p$ (FWE) } & \multicolumn{3}{|c|}{ MNI coordinates } \\
\hline & & & & & $\mathrm{X}$ & $\mathrm{Y}$ & $\mathrm{Z}$ \\
\hline \multicolumn{8}{|l|}{ MC and MI blocks } \\
\hline $\begin{array}{l}\text { Intraparietal } \\
\text { sulcus }\end{array}$ & $\mathrm{R}$ & 220 & 6.30 & $<0.001$ & 28 & -64 & 54 \\
\hline $\begin{array}{l}\text { Intraparietal } \\
\text { sulcus }\end{array}$ & $\mathrm{L}$ & 305 & 5.73 & 0.002 & -24 & -58 & 46 \\
\hline \multicolumn{8}{|l|}{ MC block } \\
\hline $\begin{array}{l}\text { Intraparietal } \\
\text { sulcus }\end{array}$ & $\mathrm{R}$ & 1755 & $>8.00$ & $<0.001$ & 28 & -64 & 56 \\
\hline $\begin{array}{l}\text { Intraparietal } \\
\text { sulcus }\end{array}$ & $\mathrm{L}$ & 2317 & 7.55 & $<0.001$ & -24 & -58 & 44 \\
\hline $\begin{array}{l}\text { Inferior frontal } \\
\text { gyrus }\end{array}$ & $\mathrm{L}$ & 487 & 7.22 & $<0.001$ & -36 & 6 & 30 \\
\hline $\begin{array}{l}\text { Middle frontal } \\
\text { gyrus }\end{array}$ & $\mathrm{R}$ & 97 & 6.13 & $<0.001$ & 26 & 6 & 56 \\
\hline $\begin{array}{l}\text { Inferior } \\
\text { temporal sulcus }\end{array}$ & $\mathrm{R}$ & 53 & 6.04 & $<0.001$ & 52 & -54 & -12 \\
\hline $\begin{array}{l}\text { Middle frontal } \\
\text { gyrus }\end{array}$ & $\mathrm{L}$ & 94 & 5.93 & $<0.001$ & -28 & 4 & 52 \\
\hline Cerebellum & $\mathrm{R}$ & 19 & 5.42 & 0.003 & 26 & -62 & -28 \\
\hline $\begin{array}{l}\text { Middle frontal } \\
\text { gyrus } \\
\text { (dorsolateral } \\
\text { prefrontal } \\
\text { cortex) }\end{array}$ & $\mathrm{R}$ & 21 & 5.23 & 0.008 & 48 & 40 & 26 \\
\hline $\begin{array}{l}\text { Pre- } \\
\text { supplementary } \\
\text { motor area }\end{array}$ & $\mathrm{L} / \mathrm{R}$ & 26 & 5.17 & 0.010 & 0 & 10 & 52 \\
\hline $\begin{array}{l}\text { Inferior } \\
\text { temporal sulcus }\end{array}$ & $\mathrm{L}$ & 13 & 5.13 & 0.012 & -44 & -56 & -10 \\
\hline $\begin{array}{l}\text { MI block } \\
\text { None }\end{array}$ & & & & & & & \\
\hline
\end{tabular}

that gets activated during the MC block is less extensively activated during the MI block.

3.2.2. Increased sustained control on blank trials during proactive control

Blank-trial activation was compared between MI and MC blocks (i.e., MI_blank > MC_blank) to test whether control in the MI block involves sustained activity in the same areas that were transiently activated in the MC block. Functional ROIs were obtained from the cognitive control contrast in the MC block from the active-trial analyses (i.e., MC_I > MC_C). The selection was limited to frontoparietal regions
Table 2

Peak voxel activation on incongruent (minus congruent) trials in the MC blocks contrasted with incongruent (minus congruent) trials in the MI blocks.

\begin{tabular}{|c|c|c|c|c|c|c|c|}
\hline \multirow[t]{2}{*}{ Region } & \multirow[t]{2}{*}{ Side } & \multirow{2}{*}{$\begin{array}{l}\text { Cluster } \\
\text { size }\end{array}$} & \multirow{2}{*}{$\begin{array}{l}Z- \\
\text { score }\end{array}$} & \multirow[t]{2}{*}{$p(\mathrm{FWE})$} & \multicolumn{3}{|c|}{ MNI coordinates } \\
\hline & & & & & $\mathrm{X}$ & $\mathrm{Y}$ & $\mathrm{Z}$ \\
\hline $\begin{array}{l}\text { Intraparietal } \\
\text { sulcus }\end{array}$ & $\mathrm{R}$ & 283 & 6.30 & $<0.001$ & 28 & -64 & 56 \\
\hline $\begin{array}{l}\text { Intraparietal } \\
\text { sulcus }\end{array}$ & $\mathrm{L}$ & 931 & 6.23 & $<0.001$ & -32 & -48 & 42 \\
\hline $\begin{array}{l}\text { Inferior frontal } \\
\text { gyrus }\end{array}$ & $\mathrm{L}$ & 164 & 6.20 & $<0.001$ & -38 & 6 & 28 \\
\hline $\begin{array}{l}\text { Middle frontal } \\
\text { gyrus }\end{array}$ & $\mathrm{L}$ & 25 & 5.77 & 0.001 & -28 & 6 & 52 \\
\hline $\begin{array}{l}\text { Inferior temporal } \\
\text { sulcus }\end{array}$ & $\mathrm{L}$ & 57 & 5.66 & 0.001 & -48 & -56 & -12 \\
\hline $\begin{array}{l}\text { Inferior temporal } \\
\text { sulcus }\end{array}$ & $\mathrm{R}$ & 35 & 5.60 & 0.001 & 50 & -52 & -12 \\
\hline
\end{tabular}

commonly associated to cognitive control (Braver, 2012; Corbetta and Shulman, 2002; Dosenbach et al., 2008; Nee et al., 2011; Niendam et al., 2012), in this case bilateral IPS, left IFG, bilateral MFG, right dlPFC, and left pre-SMA. The FIR functions displayed in Fig. 3 show larger activation on blank trials in MI blocks than in MC blocks at 8-10 s in the bilateral IPS, $p=0.036$, bilateral MFG, $p=0.026$ and the right dlPFC, $p=0.042$. This demonstrates that in these areas, control is exerted via increased activation between trials, in anticipation of a potentially incongruent trial.

In addition, we explored blank-trial activation on the whole-brain level. Blank-trial activity averaged across time bins 3 to 7 (i.e., 4-12 s post blank-onset) revealed no activation in either direction (i.e., MI_blank > MC_blank or MC_blank > MI_blank). Contrasting blank activation between MI and MC blocks on each of the five time bins individually also did not show activation in either direction.

\section{Discussion}

Using a paradigm that allowed differentiation of on-trial and intertrial (i.e., blank) activation, we showed a temporal dissociation in cognitive control, depending on the proportion congruency context (MC or MI). A fronto-parietal network comprising the bilateral IPS, left IFG, bilateral MFG, left pre-SMA, and right dlPFC showed increased transient activity on active trials in MC blocks, demonstrating the transient or reactive nature of cognitive control in this context. Conversely, activity was increased in IPS, MFG, and dlPFC during blank trials in the MI block, signaling the sustained or proactive nature of cognitive control in this

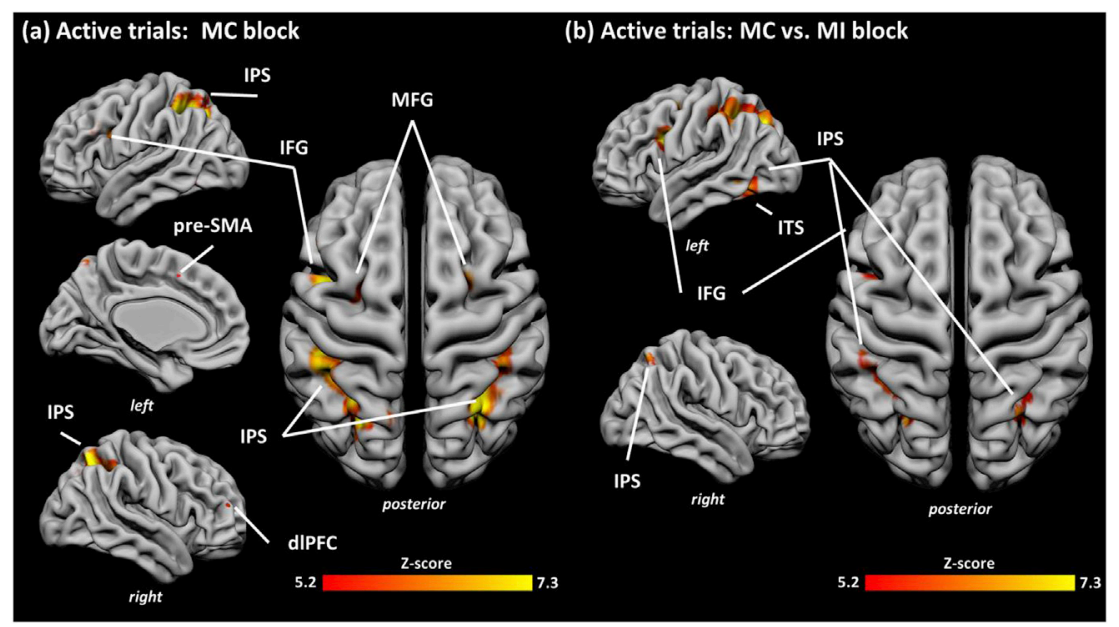

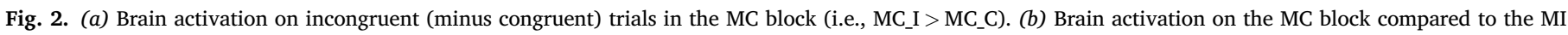

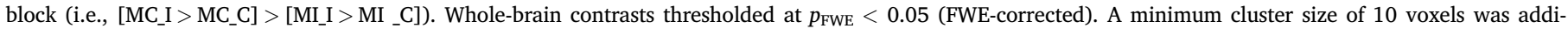
tionally imposed. 

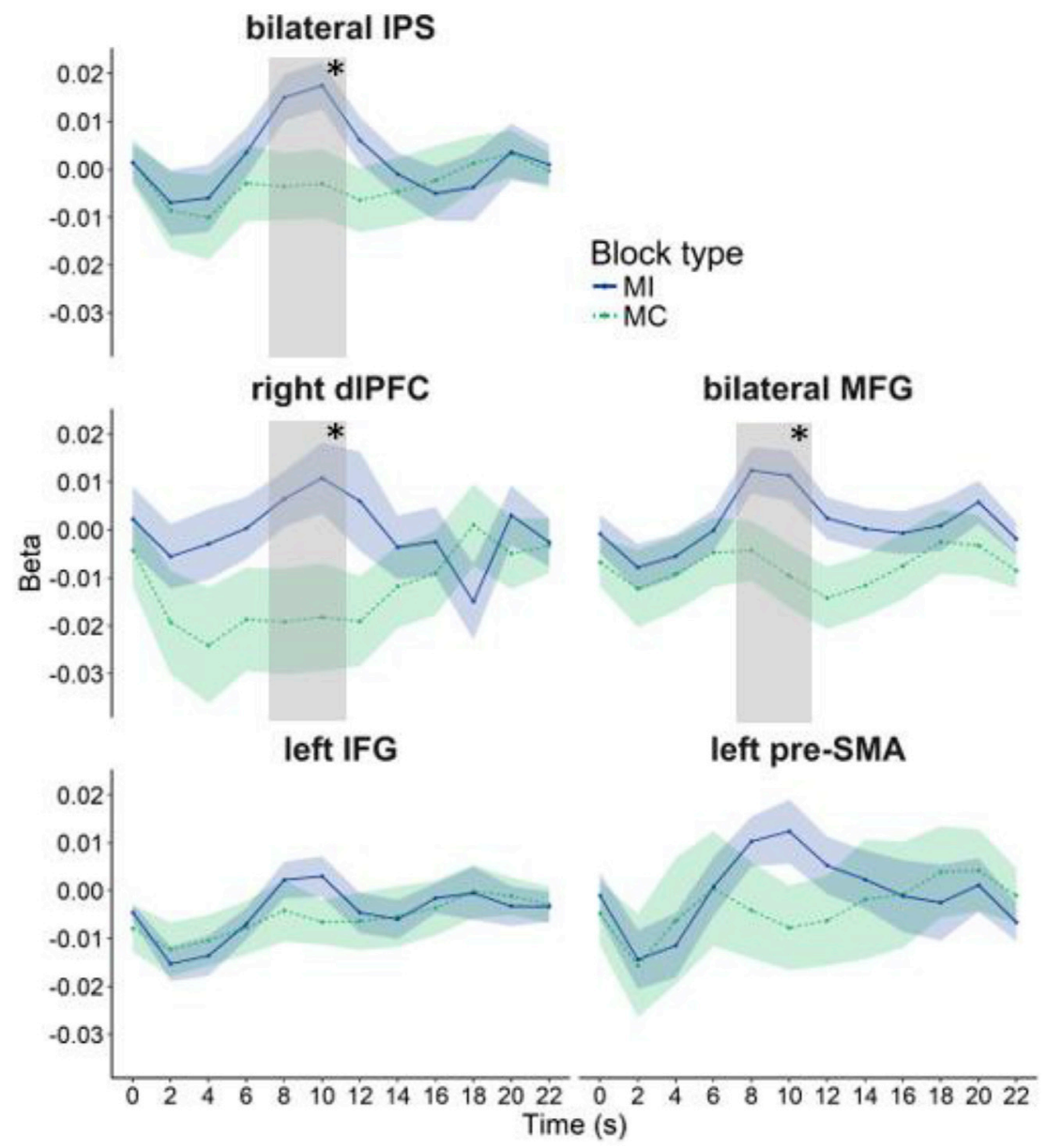

Fig. 3. Finite Impulse Response (FIR) functions showing activation during blank trials in MI and MC blocks. ${ }^{*} p<0.05$.

context. This dissociation shows that reactive and proactive control involve similar brain regions but have different temporal profiles.

\subsection{Reactive control is mirrored in transient engagement of task-relevant areas}

Several frameworks predict that cognitive control can operate on different time scales. For example, enhanced transient cortical responses are proposed to signify the dominance of reactive over proactive control on a given trial (Braver, 2012). In agreement with this, we found increased brain activity on rare incongruent trials in MC contexts, compared to frequent incongruent trials in MI contexts, attesting to the existence of a transient control mode, or reactive control.

Activated areas included the bilateral IPS and right dlPFC, two key areas of the fronto-parietal attention network (Corbetta and Shulman, 2002). Both areas have a well-established role in control of attention (Corbetta and Shulman, 2002; Niendam et al., 2012; Zhang et al., 2017) and play a central role in cognitive control (Kerns et al., 2004; MacDonald et al., 2000), for example through representation of task goals or stimulus-response rules in dlPFC (Braver, 2012; Cole and Schneider, 2007; Corbetta and Shulman, 2002). Increased activation was also found in frontal gyri (IFG, MFG) known to be involved in orienting attention (Marini et al., 2016; Walsh et al., 2011; Zhang et al., 2017), and pre-SMA, a region extensively linked to detection and resolution of conflict (Horga et al., 2011; Isoda and Hikosaka, 2007; Nachev et al., 2005).

Together, the active-trial results illuminate a network of brain regions most likely involved in detecting the occasional need for control (preSMA), reactivation of the task goal (dlPFC) and attentional orienting to the task-relevant information (parietal, midfrontal areas) on infrequent incongruent flanker trials. Given that this pattern was observed for incongruent trials in the MC context but not the MI context, it appears that the reignition of task-relevant brain areas is stronger when cognitive control is unexpectedly or infrequently required than when it is needed on most trials. This is consistent with previous work that showed increased on-trial cortical responses during reactive control on other cognitive control tasks (e.g., Carter et al., 2000; De Pisapia and Braver, 2006; Grandjean et al., 2012; Jaspar et al., 2016; Marini et al., 2016; Wilk et al., 2012).

Unexpectedly, no incongruency-related dorsal ACC activity was found, even though dorsal ACC has been extensively linked to cognitive control and/or cognitive effort (Botvinick et al., 2001; Braver, 2012; Cole and Schneider, 2007; De Pisapia and Braver, 2006; Holroyd and Yeung, 2012; Shenhav et al., 2013; Wilk et al., 2012). Yet, absence of dorsal ACC activity is not uncommon when flanker tasks are used to target cognitive control. For example, Marini et al. (2016) found no incongruency-related activity in whole-brain analyses, and a meta-analysis of different cognitive control tasks has shown that dorsal ACC is not always among the regions typically activated on flanker tasks (Nee et al., 2007). It is also relevant to mention that because of their anatomical proximity and often concurrent activation, it can be difficult to distinguish activation of dorsal ACC and pre-SMA during cognitive processing (Jahn et al., 2016).

\subsection{Intertrial activation shows the sustained nature of proactive control}

Different transient activation dynamics in fronto-parietal areas on MC and MI contexts do not directly attest to the proactive nature of control in 
the latter context. The activation during intertrial intervals however does provide support on this matter, by showing that task-relevant areas are more active on blank trials in the MI context than in the MC context. We observed that the same areas that are recruited reactively on active trials in MC contexts (i.e., bilateral IPS, bilateral MFG, and right dlPFC) are more strongly activated on blank trials in MI compared to MC contexts. The fact that the direction of the effect is opposite for active and blank trials and the fact that previous-trial congruency was controlled for, rule out the possibility that blank-trial activation reflects residual activation induced by the previous active trial. In addition, these results were obtained while experimentally controlling for visual stimulation, stimulusresponse contingencies, trial difficulty, motor response, accuracy, and response time.

Diminished transient responses to incongruency during the MI context and increased sustained responses between trials in that same context offer an empirical demonstration of the mechanisms involved in reactive and proactive control. As suggested before (De Pisapia and Braver, 2006; Kerns et al., 2004), a large proportion incongruency may lead to a tonic increase of cognitive control-related activity, which in turn reduces the transient neural response to incongruency at the trial level. This also explains the smaller behavioural effect of incongruency in MI contexts.

The brain areas that appear to be involved in this balance of transient and sustained activity included IPS and dIPFC. These areas are part of the fronto-parietal network that serves to initiate and adjust cognitive control (Corbetta and Shulman, 2002; Dosenbach et al., 2008) and encompasses functions such as sustaining representations of the task goal (e.g., "respond to central number"; MacDonald et al., 2000; Miller and Cohen, 2001; Braver, 2012) and attentional engagement (e.g., narrowing attention to central number; Walsh et al., 2011; Marini et al., 2016). Since dlPFC and IPS were also recruited during reactive control, task goal representation and attentional engagement may be active during both control modes in a qualitatively similar way but, importantly, only transiently when control is occasionally needed during reactive control.

The involvement of similar areas is consistent with findings of hybrid fMRI studies that statistically separated transient and sustained responses to show involvement of similar areas in reactive and proactive control (Braver et al., 2003; Marini et al., 2016). However, other work has shown that transient and sustained control may recruit different areas (e.g., Dosenbach et al., 2008; Wilk et al., 2012). Since the current whole-brain analyses did not show any blank-trial activation, it remains speculative whether context-driven reactive and proactive control are indeed solely bound to similar brain areas and differ only in terms of temporal profiles, or whether they are also partly comprised by different regions.

The fact that sustained activation extended beyond dlPFC to IPS is partly consistent with the work of Dosenbach et al. (2008). They suggest that both dlPFC and IPS have a superordinate function in maintenance of task goals and contexts, but on a short time scale (i.e., in the range of a small number of trials), while ACC and anterior insula provide stable task-set maintenance (i.e., in a range that spans all trials in a task epoch (see also Holroyd and Yeung, 2012). The current results partly agree with this dual-networks perspective in the sense that dlPFC and IPS indeed showed transient trial-to-trial adjustments. However, both regions were also activated in a sustained manner. This suggests that dlPFC and IPS are functionally related and can operate transiently, but also on a time scale that spans more than a few trials (i.e., on the block level). Inconsistent with Dosenbach et al. (2008), we found no activation of ACC or anterior insula on a long time scale. In fact, these regions appeared not to carry any control signal in the current task. The current results therefore cannot elucidate if and how ACC and anterior insula fit into dual-networks perspectives on cognitive control. Finally, given its extensive involvement in attentional orienting (Corbetta and Shulman, 2002; Marini et al., 2016; Walsh et al., 2011), the role of IPS in top-down control may be qualitatively different from more abstract task-goal maintenance.

\subsection{Activity-silent cognitive control}

The exact implementation of transient and sustained engagement of task-relevant areas in reactive and proactive control remains an open question. A potential explanation may come from working memory studies that have demonstrated that recent information can remain dormant in pre-synaptic (activity-silent) connectivity. When needed, such connectivity may suddenly lead to (metabolically more costly) spiking activity (Mongillo et al., 2008; Rose et al., 2016; Stokes, 2015). In line with this synaptic working memory theory, we suggest that reactive control in MC contexts involves maintaining task-relevant information in pre-synaptic traces. When a task cue or stimulus is presented (in this case a rare incongruent trial) such traces can be reactivated, leading to a transient rise of fronto-parietal activation and a reconnection of this network with relevant sensory areas.

Further in line with synaptic working memory theory, we suggest that in proactive control mode, task-relevant information remains in active firing mode because it needs to be applied frequently. This has the advantage of being more readily available at all times, but also increases metabolic costs. Sustained control therefore taxes the cognitive system (Braver et al., 2007), and subjects will tend to avoid it to reduce cognitive effort (Kool et al., 2010).

In the current design, sustaining control was necessary to achieve optimal task performance in MI blocks. Subjects could expect the next trial to be incongruent but the occurrence of the next stimulus could not be predicted because of the jittered stimulus presentation. This forced subjects to remain on guard in the intertrial interval in the MI block. Elaborating on synaptic working memory and effort avoidance theories, one would expect this strategy to be abandoned in favour of a more metabolically efficient (i.e., less effortful) strategy when stimulus presentation is temporally predictable. In that case, activity may stay silent throughout most of the interval also in MI blocks, and ramp-up right before stimulus presentation, resulting in a phasic form of proactive control. This prediction remains to be tested.

\subsection{Conclusion}

The current study demonstrates how the brain flexibly implements cognitive control on different time scales, depending on the context. It shows that cognitive control in MC and MI contexts is subtended by different temporal activation of the same fronto-parietal areas. In MC contexts, or reactive control mode, transient activity in task-relevant fronto-parietal areas is larger. In MI contexts, or proactive control mode, sustained activation in similar areas is increased between trials. Together, these results explain how reactive and proactive control recruit similar brain areas but on different time scales.

\section{Declarations of interest statement}

None.

\section{Acknowledgements}

This work was supported by the Research Foundation Flanders, Belgium (FWO Vlaanderen; FWOTM765 to B.A. and FWOAL685 to T.V. and E.V.d.B).

\section{References}

Botvinick, M.M., Braver, T.S., Barch, D.M., Carter, C.S., Cohen, J.D., 2001. Conflict monitoring and cognitive control. Psychol. Rev. 108 (3), 624-652. https://doi.org/ 10.1037/0033-295X.108.3.624.

Braver, T.S., 2012. The variable nature of cognitive control: a dual mechanisms framework. Trends Cognit. Sci. 16 (2), 106-113. https://doi.org/10.1016/j.tics. 2011.12.010.

Braver, T.S., Gray, J.R., Burgess, G.C., 2007. Explaining the Many Varieties of Working Memory Variation: Dual Mechanisms of Cognitive Control. In: Conway, A.A.R., Jarrold, C., Kane, M.J., Miyake, A., Towse, J.N. (Eds.), Variation in Working Memory. 
Oxford University Press, Oxford, pp. 1-79. https://doi.org/10.3758/s13423-0110165-y.

Braver, T.S., Reynolds, J.R., Donaldson, D.I., 2003. Neural mechanisms of transient and sustained cognitive control during task switching. Neuron 39, 713-726. https://doi. org/10.1016/S0896-6273(03)00466-5.

Bugg, J., Crump, M.J.C., 2012. In support of a distinction between voluntary and stimulus-driven control: a review of the literature on proportion congruent effects. Front. Psychol. 3, 1-16. https://doi.org/10.3389/fpsyg.2012.00367.

Carter, C.S., Macdonald, A.M., Botvinick, M., Ross, L.L., Stenger, A., Noll, D., et al., 2000 Parsing functions executive of the Strategic processes: cortex cingulate anterior evaluative. Proc. Natl. Acad. Sci. U.S.A. 97 (4), 1944-1948. Retrieved from. http:// www.pubmedcentral.nih.gov/articlerender.fcgi? artid $=26541$ \&tool $=$ pmcentrez\&rendertype $=$ abstract

Cole, M.W., Schneider, W., 2007. The cognitive control network: Integrated cortical regions with dissociable functions. Neuroimage 37 (1), 343-360. https://doi.org/10. 1016/j.neuroimage.2007.03.071.

Corbetta, M., Shulman, G.L., 2002. Control of goal-directed and stimulus-driven attention in the brain. Nat. Rev. Neurosci. 3 (3), 201-215. https://doi.org/10.1038/nrn755.

De Pisapia, N., Braver, T.S., 2006. A model of dual control mechanisms through anterior cingulate and prefrontal cortex interactions. Neurocomputing 69 (10-12), 1322-1326. https://doi.org/10.1016/j.neucom.2005.12.100.

Dosenbach, N.U.F., Fair, D.A., Cohen, A.L., Schlaggar, B.L., Petersen, S.E., 2008. A dualnetworks architecture of top-down control. Trends Cognit. Sci. 12 (3), 99-105. https://doi.org/10.1016/j.tics.2008.01.001.

Eriksen, B.A., Eriksen, C.W., 1974. Effects of noise letters upon the identification of a target letter in a nonsearch task. Percept. Psychophys. 16 (1), 143-149.

Grandjean, J., D'Ostilio, K., Phillips, C., Balteau, E., Degueldre, C., Luxen, A., et al., 2012. Modulation of brain activity during a Stroop inhibitory task by the kind of cognitive control required. PLoS One 7 (7), e41513. https://doi.org/10.1371/journal.pone. 0041513.

Grinband, J., Savitskaya, J., Wager, T.D., Teichert, T., Ferrera, V.P., Hirsch, J., 2011. The dorsal medial frontal cortex is sensitive to time on task, not response conflict or error likelihood. Neuroimage 57 (2), 303-311. https://doi.org/10.1016/j.neuroimage. 2010.12.027.

Holroyd, C.B., Yeung, N., 2012. Motivation of extended behaviors by anterior cingulate cortex. Trends Cognit. Sci. 16 (2), 122-128. https://doi.org/10.1016/j.tics.2011.12. 008.

Horga, G., Maia, T.V., Wang, P., Wang, Z., Marsh, R., Peterson, B.S., 2011. Adaptation to conflict via context-driven anticipatory signals in the dorsomedial prefrontal cortex. J. Neurosci.: Off. J. Soc. Neurosci. 31 (45), 16208-16216. https://doi.org/10.1523/ JNEUROSCI.2783-11.2011.

Isoda, M., Hikosaka, O., 2007. Switching from automatic to controlled action by monkey medial frontal cortex. Nat. Neurosci. 10 (2), 240-248. https://doi.org/10.1038/ nn1830.

Jahn, A., Nee, D.E., Alexander, W.H., Brown, J.W., 2016. Distinct Regions within Medial Prefrontal Cortex Process Pain and Cognition. J. Neurosci. 36 (49), 12385-12392. https://doi.org/10.1523/JNEUROSCI.2180-16.2016.

Jaspar, M., Manard, M., Dideberg, V., Bours, V., Maquet, P., Collette, F., 2016. Influence of COMT Genotype on Antero-posterior Cortical Functional Connectivity Underlying Interference Resolution. Cerebr. Cortex 26 (2), 498-509. https://doi.org/10.1093/ cercor/bhu188.

Jiang, J., Heller, K., Egner, T., 2014. Bayesian modeling of flexible cognitive control. Neurosci. Biobehav. Rev. 1-14. https://doi.org/10.1016/j.neubiorev.2014.06.001.

Kerns, J.G., Cohen, J.D., MacDonald, A.W., Cho, R.Y., Stenger, V.A., Carter, C.S., 2004 Anterior cingulate conflict monitoring and adjustments in control. Science (New York, N.Y.) 303 (5660), 1023-1026. https://doi.org/10.1126/science.1089910.

Kool, W., McGuire, J.T., Rosen, Z.B., Botvinick, M.M., 2010. Decision making and the avoidance of cognitive demand. J. Exp. Psychol. Gen. 139 (4), 665-682. https://doi. org/10.1037/a0020198.

Logan, G.D., Zbrodoff, N.J., 1979. When it helps to be misled: Facilitative effects of increasing the frequency of conflicting stimuli in a Stroop-like task. Mem. Cognit. 7 (3), 166-174. https://doi.org/10.3758/BF03197535.

MacDonald, a W., Cohen, J.D., Stenger, V. a, Carter, C.S., 2000. Dissociating the role of the dorsolateral prefrontal and anterior cingulate cortex in cognitive control. Science (New York, N.Y.) 288. June, 1835-1838. https://doi.org/10.1126/science.288.5472. 1835.

Marini, F., Demeter, E., Roberts, K.C., Chelazzi, L., Woldorff, M.G., 2016. Orchestrating Proactive and Reactive Mechanisms for Filtering Distracting Information: BrainBehavior Relationships Revealed by a Mixed-Design fMRI Study. J. Neurosci. 36 (3), 988-1000. https://doi.org/10.1523/JNEUROSCI.2966-15.2016.

Maris, E., Oostenveld, R., 2007. Nonparametric statistical testing of EEG- and MEG-data J. Neurosci. Methods 164 (1), 177-190. https://doi.org/10.1016/j.jneumeth.2007. 03.024.
Miller, E.K., Cohen, J.D., 2001. An Integrative Theory Of Prefrontal Cortex Function. Annu. Rev. Neurosci. 24, 167-202.

Mongillo, G., Barak, O., Tsodyks, M., 2008. Synaptic theory of working memory. Science 319, 1543-1546. https://doi.org/10.1126/science.1150769.

Nachev, P., Rees, G., Parton, A., Kennard, C., Husain, M., 2005. Volition and Conflict in Human Medial Frontal Cortex. Curr. Biol. 15, 122-128.

Nee, D.E., Kastner, S., Brown, J.W., 2011. Functional heterogeneity of conflict, error, taskswitching, and unexpectedness effects within medial prefrontal cortex. Neuroimage 54 (1), 528-540. https://doi.org/10.1016/j.neuroimage.2010.08.027.

Nee, D.E., Wager, T.D., Jonides, J., 2007. Interference resolution: insights from a metaanalysis of neuroimaging tasks. Cognit. Affect Behav. Neurosci. 7 (1), 1-17. https:// doi.org/10.3758/CABN.7.1.1.

Niendam, T.A., Laird, A.R., Ray, K.L., Dean, Y.M., Glahn, D.C., Carter, C.S., 2012. Metaanalytic evidence for a superordinate cognitive control network subserving diverse executive functions. Cognit. Affect Behav. Neurosci. 12 (2), 241-268. https://doi. org/10.3758/s13415-011-0083-5.

Olsen, A., Brunner, J.F., Anne, K., Evensen, I., Garzon, B., Landrø, N.I., Håberg, A.K., 2010. The Functional Topography and Temporal Dynamics of Overlapping and Distinct Brain Activations for Adaptive Task Control and Stable Task-set Maintenance during Performance of an fMRI-adapted Clinical Continuous Performance Test. J. Cognit. Neurosci. 25 (6), 903-919. https://doi.org/10.1162/jocn.

Petersen, S.E., Dubis, J.W., 2012. The mixed block/event-related design. Neuroimage 62 (2), 1177-1184. https://doi.org/10.1016/j.neuroimage.2011.09.084.

Poldrack, R.A., Mumford, J.A., Nichols, T.E., 2011. Handbook of functional MRI data analysis, vol. 4. Cambridge University Press, New York.

Psychology Software Tools, 2012. E-Prime 2.0. Psychology Software Tools, Inc. http:// www.pstnet.com.

Ridderinkhof, K.R., 2002. Micro- and macro-adjustments of task set: Activation and suppression in conflict tasks. Psychol. Res. 66 (4), 312-323. https://doi.org/10. 1007/s00426-002-0104-7.

Rose, N.S., LaRocque, J.J., Rigall, A.C., Gosseries, O., Starett, M.J., Meyering, E.E., Postle, B.R., 2016. Reactivation of latent working memories with transcranial magnetic stimulation. Science 354 (6316), 1136-1139. https://doi.org/10.1126/ science.aah7011.

Schmidt, J.R., Besner, D., 2008. The Stroop effect: why proportion congruent has nothing to do with congruency and everything to do with contingency. J. Exp. Psychol. Learn. Mem. Cognit. 34 (3), 514-523. https://doi.org/10.1037/0278-7393.34.3.514.

Seeley, W.W., Menon, V., Schatzberg, A.F., Keller, J., Glover, G.H., Kenna, H., et al., 2007. Dissociable Intrinsic Connectivity Networks for Salience Processing and Executive Control. J. Neurosci. 27 (9), 2349-2356. https://doi.org/10.1523/JNEUROSCI.558706.2007.

Shenhav, A., Botvinick, M.M., Cohen, J.D., 2013. The expected value of control: An integrative theory of anterior cingulate cortex function. Neuron 79 (2), 217-240. https://doi.org/10.1016/j.neuron.2013.07.007.

Shenhav, A., Musslick, S., Lieder, F., Kool, W., Griffiths, T.L., Cohen, J.D., Botvinick, M.M., 2017. Toward a Rational and Mechanistic Account of Mental Effort. Annu. Rev. Neurosci. 40 (1), 99-124. https://doi.org/10.1146/annurev-neuro072116-031526.

Stokes, M.G., 2015. 'Activity-silent' working memory in prefrontal cortex: a dynamic coding framework. Trends Cognit. Sci. 19 (7), 394-405. https://doi.org/10.1016/j. tics.2015.05.004.

Stroop, J.R., 1935. Studies of interference in serial verbal reactions. J. Exp. Psychol. 18 (6), 643-662.

Vassena, E., Silvetti, M., Boehler, C.N., Achten, E., Fias, W., Verguts, T., 2014. Overlapping neural systems represent cognitive effort and reward anticipation. PLoS One 9 (3). https://doi.org/10.1371/journal.pone.0091008.

Verguts, T., Vassena, E., Silvetti, M., 2015. Adaptive effort investment in cognitive and physical tasks: a neurocomputational model. Front. Behav. Neurosci. 9. March. https://doi.org/10.3389/fnbeh.2015.00057.

Visscher, K.M., Miezin, F.M., Kelly, J.E., Buckner, R.L., Donaldson, D.I., McAvoy, M.P., et al., 2003. Mixed blocked/event-related designs separate transient and sustained activity in fMRI. Neuroimage 19 (4), 1694-1708. https://doi.org/10.1016/S10538119(03)00178-2.

Walsh, B.J., Buonocore, M.H., Carter, C.S., Mangun, G.R., 2011. Integrating Conflict Detection and Attentional Control Mechanisms. J. Cognit. Neurosci. 23 (9), 2191-2201. https://doi.org/10.1162/jocn.2010.21595.

Wilk, H.A., Ezekiel, F., Morton, J.B., 2012. Brain regions associated with moment-tomoment adjustments in control and stable task-set maintenance. Neuroimage 59 (2), 1960-1967. https://doi.org/10.1016/j.neuroimage.2011.09.011.

Zhang, R., Geng, X., Lee, T.M.C., 2017. Large-scale functional neural network correlates of response inhibition: an fMRI meta-analysis. Brain Struct. Funct. 222 (9), 1-18. https://doi.org/10.1007/s00429-017-1443-x. 